\title{
Molecular evolution and expression divergence of three key Met biosynthetic genes in plants: CGS, HMT and MMT
}

\author{
Man Zhao ${ }^{\text {Corresp.. }}{ }^{1}$, Wenyi Wang ${ }^{1}$, Lei Wei ${ }^{1}$, Peng Chen ${ }^{1}$, Fengjie Yuan ${ }^{2}$, Zhao Wang ${ }^{1}$, Xiangxian Ying \\ ${ }^{1}$ College of Biotechnology and Bioengineering, Zhejiang University of Technology, Hangzhou, China \\ 2 Institute of Crop Science, Zhejiang Academy of Agricultural Sciences, Hangzhou, China \\ Corresponding Author: Man Zhao \\ Email address: mzhao@zjut.edu.cn
}

Methionine (Met) is an essential sulfur-containing amino acid in animals. Cereal and legume crops with limiting levels of Met represent the major food and feed sources for animals. In plants, cystathionine $\gamma$-synthase (CGS), methionine methyltransferase (MMT) and homocysteine methyltransferase (HMT) are committing enzymes synergistically synthesizing Met through the aspartate (Asp) family pathway and the S-methylmethionine ( SMM) cycle. The biological functions of CGS, MMT and HMT genes have been respectively studied, whereas their evolution patterns and their contribution to the evolution of Met biosynthetic pathway in plants are unknown. In the present study, to reveal their evolution patterns and contribution, the evolutionary relationship of CGS, MMT and HMT gene families were reconstructed. The results showed that MMTs emerged since the ancestors of the land plants and kept conserved during evolution, while the CGSs and HMTs had diverged. The CGS genes were divided into two branches in the angiosperms, Class 1 and Class 2, of which Class 2 only contained the grasses. However, the HMT genes diverged into Class 1 and Class 2 in all of the seed plants. Further, the gene structure analysis revealed that the CGSs, MMTs and HMTs were relatively conserved except for the CGSs in Class 2. According to the expression of CGS, HMT and MMT genes in soybeans, as well as in the database of soybean, rice and Arabidopsis, the expression patterns of the MMTs are showed to be consistently higher in leaves than in seeds. However, the expression of CGSs and HMTs had diverged, either higher expressed in leaves or seeds, or showing the fluctuated expression. Besides, the functions of HMT genes had diverged into the repair of $S$-adenosylmethionine ( $(R, S)$-AdoMet) and SMM catabolism during the evolution. The results indicated that the CGS and HMT genes have occurred partial subfunctionalization. Finally, given the evolution and expression of the CGS, HMT and MMT gene families, we built the evolutionary model of the Met biosynthetic pathways in plants. The model proposed that the Asp family pathway existed in all the plant lineages, while the SMM cycle emerged since the ancestor of land plants and then diverged since the ancestor of 
seed plants. The model suggested that the evolution of Met biosynthetic pathway is basically consistent with that of plants, which might be vital to the growth and development of different botanical lineages during the evolution. 
Research article

2

3 Molecular evolution and expression divergence of three key Met biosynthetic genes in plants: CGS, HMT and $M M T$

5

6 Man Zhao ${ }^{1, *}$, Wenyi Wang ${ }^{1}$, Lei Wei ${ }^{1}$, Peng Chen ${ }^{1}$, Fengjie Yuan ${ }^{2}$, Zhao Wang ${ }^{1}$, Xiangxian

7 Ying ${ }^{1, *}$

81 College of Biotechnology and Bioengineering, Zhejiang University of Technology, 310014

9 Hangzhou, China;

102 Institute of Crop Science, Zhejiang Academy of Agricultural Sciences, 310014 Hangzhou, 11 China

12 *Correspondence: mzhao@zjut.edu.cn (M.Z.). yingxx@,zjut.edu.cn (X.Y.)

\section{Author email list}

15 M.Z.: mzhao@zjut.edu.cn

16 W.W.: hzwywang@163.com

17 L.W.: leiweistudy@163.com

18 P.C.: chenpengoffice@163.com

19 F. Y.: fjyuanhz@126.com

20 Z.W.: hzwangzhao@163.com

21 X.Y.: yingxx@zjut.edu.cn

\section{ABSTRACT}

Methionine (Met) is an essential sulfur-containing amino acid in animals. Cereal and legume crops with limiting levels of Met represent the major food and feed sources for animals. In plants, cystathionine $\gamma$-synthase (CGS), methionine methyltransferase (MMT) and homocysteine methyltransferase (HMT) are committing enzymes synergistically synthesizing Met through the aspartate (Asp) family pathway and the S-methylmethionine (SMM) cycle. The biological functions of $C G S, M M T$ and $H M T$ genes have been respectively studied, whereas their evolution patterns and their contribution to the evolution of Met biosynthetic pathway in plants are unknown. In the present study, to reveal their evolution patterns and contribution, the 
32 evolutionary relationship of $C G S, M M T$ and $H M T$ gene families were reconstructed. The results

33 showed that MMTs emerged since the ancestors of the land plants and kept conserved during

34 evolution, while the CGSs and HMTs had diverged. The CGS genes were divided into two

35 branches in the angiosperms, Class 1 and Class 2, of which Class 2 only contained the grasses.

36 However, the HMT genes diverged into Class 1 and Class 2 in all of the seed plants. Further, the gene structure analysis revealed that the CGSs, MMTs and HMTs were relatively conserved except for the CGSs in Class 2. According to the expression of CGS, HMT and MMT genes in soybeans, as well as in the database of soybean, rice and Arabidopsis, the expression patterns of the $M M T$ s are showed to be consistently higher in leaves than in seeds. However, the expression of CGSs and HMTs had diverged, either higher expressed in leaves or seeds, or showing the fluctuated expression. Besides, the functions of $H M T$ genes had diverged into the repair of $S$ adenosylmethionine $((R, S)$-AdoMet) and SMM catabolism during the evolution. The results indicated that the $C G S$ and HMT genes have occurred partial subfunctionalization. Finally, given the evolution and expression of the CGS, HMT and MMT gene families, we built the evolutionary model of the Met biosynthetic pathways in plants. The model proposed that the Asp family pathway existed in all the plant lineages, while the SMM cycle emerged since the ancestor of land plants and then diverged since the ancestor of seed plants. The model suggested that the evolution of Met biosynthetic pathway is basically consistent with that of plants, which might be vital to the growth and development of different botanical lineages during the evolution.

\section{INTRODUCTION}

Met is an essential amino acid, which is mainly obtained from human and animal foods. Met plays important functions, not only as a protein component and in the initiation of mRNA translation, but also in indirectly regulating various metabolic processes through its main catabolic product, $S$-adenosylmethionine (SAM, AdoMet) (Galili \& Amir, 2013; Roje, 2006; Sauter et al., 2013). Despite its important functions, Met could only be synthesized in plants and microorganisms (Galili \& Amir, 2013).

The biosynthetic pathway of Met has been widely studied, which could be synthesized by 
62

63

64

65

66

67

68

69

70

steps from homoserine: homoserine - O-succinylhomoserine - cystathionine - homocysterine methionine, which are catalyzed by homoserine O-succinyltransferase, cystathionine gamasynthase, cystathionine beta-lyase and methionine synthase, respectively (Ferla and Patrick, 2014). However, in Corynebacterium glutamicum, O-succinylhomoserine is replaced by Oacetylhomoserine. Besides, there is another pathway from $\mathrm{O}$-acetylhomoserine direct to methionine catalyzed by O-acetylhomoserine Sulfhydrylase (Bolten et al., 2010; Willke, 2014).

In plants, Met can be synthesized through the Asp family pathway as well as the SMM cycle. In the Asp family pathway process, homoserine is firstly converted into Ophosphohomoserine (OPH) by homoserine kinase (HSK). Then, the condensation reaction of OPH with cysteine is catalyzed into cystathionine by cystathionine gamma-synthase (CGS), and cystathionine is hydrolyzed into homocysteine through cystathionine beta-lyase. Next, Met is synthesized de novo through methionine synthase (Datko et al., 1974). As for the SMM cycle, MMT uses Met, synthesized by the Asp family pathway, and SAM to form SMM, then SMM and homocysteine (Hcy) are converted into two molecules of Met through the catalysis of HMT (Bourgis et al., 1999; Ranocha et al., 2008; Lee et al., 2008; Cohen et al., 2017a).

Furthermore, the biosynthesis process of Met during the development of plants is revealed by genetic and biochemical experiments. First, Met is synthesized by the Asp family pathway in rosette leaves, in which it is converted into SMM by MMT; second, the SMM is translocated into reproductive tissues, such as siliques and seeds, and reconverted back into Met in the developing seeds by HMT (Bourgis et al., 1999; Ranocha et al., 2001; Lee et al., 2008; Cohen et al., 2017b; Kocsis et al., 2003). Additionally, the possible contribution of SMM to the stress effects was proposed (Cohen et al., 2017b). Above all, CGS, HMT and MMT are essential enzymes in the synthesis of Met.

CGS is the mainly regulatory enzyme in the Asp family pathway. In Arabidopsis, when CGS was constitutively over-expressed, the soluble Met and SMM accumulated in specific stages, such as flowers, siliques, seedling tissues and roots of mature plants (Kim et al., 2002). Contrarily, the repression of CGSs made the plants abnormal and produced partial methionine auxotrophy (Kim \& Leustek, 2000). Interestingly, when the seed-specific repression of $C G S$ was performed, more SMMs were transported from the leaves to reproductive organs, in which there were higher reconversion rates of SMM to Met, and more Met was accumulated in seeds (Cohen et al., 2017b). In addition, studies have reported that the expressions of $C G S$ were in the negative 
93 feedback regulation of their products, Met or SAM, in wild-type Arabidopsis (Kim \& Leustek, 94 2000; Thompson et al., 1982; Ranocha et al., 2000). Further, the MTO1 region in the first exon 95 of $A t C G S$ was proved to result in its negative feedback regulation (Chiba et al., 1999; Ominato et 96 al., 2002). In mtol mutants of AtCGS1, both the enzyme levels and soluble Met levels were 97 increased (Chiba et al., 1999). Afterwards, the seed-specific expression of the feedback98 insensitive form of AtCGSs in plants were also studied, but with different results (Cohen et al., 99 2017b; Hanafy et al., 2013; Song et al., 2013; Cohen et al., 2016; Matityahu et al., 2013; Cohen 100 et al., 2014). For example, in Arabidopsis, soybean and tobacco, the sulfur-associated metabolism was altered and the soluble Met was significantly elevated in seeds. However, there

102 was no Met increase in azuki bean (Matityahu et al., 2013). Therefore, the CGS gene family might have diverged in different organisms during evolution.

HMT and MMT are essential in the SMM cycle (Cohen et al., 2017a; Zhao et al., 2018). The evolution and expression of HMTs have been studied, as detailed in our previous research. Research found that HMTs have diverged into two clades in seed plants and that their expression also diverged. It has been proposed that the divergence of HMTs might be crucial to meeting the needs of plant development and growth (Zhao et al., 2018). As for MMT, it was only studied in Arabidopsis by catalyzing the synthesis of SMM from Met and AdoMet (Ranocha, 2001). Nevertheless, the systematic evolution patterns of the three key enzymes CGSs, HMTs and MMTs in plants, and how they contribute to the evolution of the Met biosynthesis pathway are unclear.

Soybean is an important economic crop, as it is a source of vegetable proteins in the human

114 diet. In soybean seeds, major storage proteins consist of glycinin (11S) and conglycinin (7S), and

115 11S proteins account for approximately 30\% (Nielsen et al., 1989; Harada et al., 1989). The

116 sulfur-containing methionine is an essential amino acid, the level of which often limits the

117 nutritional value of crop plants (Galili et al. 2005). Therefore, considering the importance of the

118 three genes CGS, MMT and HMT in the synthesis of Met and soybeans, this study

119 comprehensively analyzed their evolutionary history, including their phylogenetic relationship

120 and gene structures, and examined their selection pressures. Their expression profiles in

121 soybeans were also widely analyzed. Taken together, this research is helpful for understanding

122 the evolutionary history and functional divergence of the $C G S, M M T$ and $H M T$ gene families in 123 plants, and might also provide an overall picture of the evolutionary and functional model of the 
124 Met biosynthetic pathway in plants.

125

126

MATERIALS AND METHODS

\section{Phylogenetic analysis}

128 The gene sequences in full genomes of plants were examined with genes in A. thaliana as 129 query. The sequences followed the criteria: E-value $<1 \times \mathrm{e}-05$ in the BLASTN and TBLASTN

130 programs, and an amino acid identity above 40\%, which were downloaded from the databases of

131 Phytozome (http://www.phytozome.net/), congenie (http://congenie.org/) and NCBI

132 (https://www.ncbi.nlm.nih.gov/). Altogether, 49 CGS sequences and $43 M M T$ sequences were

133 obtained from the major plant lineages studied (Tables S1,S2 in File 3). Multiple alignments of

134 gene sequences were executed in the Clustal X v1.81 program with default parameters and

135 alignments, optimized via manual adjustments using BioEdit v 7.0.9.0 (Thompson et al., 1997;

136 Hall, 1999). Maximum likelihood (ML) and Neighbor-Joining (NJ) trees were reconstructed

137 using PhyML online with the GTR + G + I model and MEGA6 software (Guindon \& Gascuel,

138 2003; Tamura et al., 2013). The resultant trees were represented using MEGA 6. The

139 phylogenetic tree of HMT genes has been shown in our previous study (Zhao et al., 2018).

\section{Analysis of gene structure}

141 The intron and exon structures of $C G S$ and $M M T$ genes were analyzed according to their

142 genome sequences and coding sequences. The length and numbers of introns and exons are

143 shown in Table S3 in File S3. In addition, the conserved motifs in proteins were detected using

144 the Multiple Em for Motif Elicitation (MEME) server (http://meme-suite.org/tools/meme)

145 (Bailey et al., 2009). The server was run using the default values and choices. We conducted the

146 search for 16 motifs in proteins arbitrarily. The motifs retrieved by MEME were reported

147 according to their statistical significance, and the most statistically significant (low E-value) ones

148 were shown first. The E-value of a motif is based on its log likelihood ratio, width, sites, and the

149 size of the set. The motifs of HMTs have been analyzed in our previous results (Zhao et al.,

150 2018).

\section{Detection of selection pressures}

152 To estimate the selection pressures in the gene families, the codeml program from the

153 PAML v4.4 package, on the basis of codon sequence alignments, was performed (Yang, 2007). 
154 The likelihood ratio test (LRT) is a general method for testing assumptions (model parameters)

155 by comparing two competing hypotheses.

\section{Plant materials and growth conditions}

157 The cultivated soybean 'Chuandou 4' was grown at a farm in Fuyang (Hangzhou, China)

158 during summer. Each materials of the leaves, stems, flowers, and 2-, 3-, 4-, 5- and 6-week post-

159 fertilization fruits for the gene expression study were harvested at the same time. The harvested

160 tissues were immediately stored in liquid $\mathrm{N} 2$ and then stored at $-80{ }^{\circ} \mathrm{C}$ for total RNA extraction

161 using TRIzol reagent (Invitrogen).

\section{Realtime RT-PCR analyses}

163 Two micrograms of total RNA were used to synthesize the first strand cDNA using a

164 ReverTra Ace qPCR RT Kit cDNA Synthesis Kit (TOYOBO). Quantitative RT-PCR (qRT-PCR) 165 was conducted using a ChamQ TM SYBR qPCR Master Mix (Vazyme) in a CFX Connect Real-

166 Time system (BIO-RAD). ACTIN (Glyma.18G290800) was used as an internal control. Each

167 experiment was performed using three independent biological samples. PCR was performed in a

$16825.0 \mu \mathrm{L}$ reaction mixture containing $5 \mu \mathrm{L}$ ChamQ TM SYBR qPCR Master Mix (Vazyme), 50

169 ng cDNA template, $0.4 \mu \mathrm{L}$ of each primer $(10.0 \mu \mathrm{M})$ and $3.2 \mu \mathrm{L}$ of double distilled $\mathrm{H}_{2} \mathrm{O}$ (dd

$170 \mathrm{H}_{2} \mathrm{O}$ ). The optimized operational procedure was performed as follows: 2 min at $95^{\circ} \mathrm{C}(1 \mathrm{cycle})$,

$17110 \mathrm{~s}$ at $95{ }^{\circ} \mathrm{C}, 30 \mathrm{~s}$ at $60{ }^{\circ} \mathrm{C}(40$ cycles $), 5 \mathrm{~s}$ at $65^{\circ} \mathrm{C}$ and $5 \mathrm{~s}$ at $95{ }^{\circ} \mathrm{C}(1$ cycle for the melting

172 curve analysis). The relative gene expression was evaluated as previously described (Livak \&

173 Schmittgen, 2001).

174 The expression of genes in different tissues was analyzed in the PLEXdb database

175 (http://www.plexdb.org/index.php) (Dash et al., 2012).

\section{Promoters analysis}

177 The promoter sequences (2000 bp upstream of the transcription initiation site), GmaHMTs,

178 GmaCGSs and GmaMMTs, were obtained from Phytozome. To identify the putative cis-acting

179 regulatory elements, the promoter sequences of GmaHMTs, GmaCGSs and GmaMMTs were

180 submitted to PlantCARE (http://bioinformatics.psb.ugent.be/webtools/plantcare/html/) (Lescot et

181 al., 2002).

\section{Statistical analysis}

183 In this study, standard deviations were calculated based on a minimum of three independent 184 replicates. Comparative statistical analyses of groups were performed using Student's t test. 


\section{RESULTS}

\section{Identification and phylogenetic analysis of $C G S, M M T$ and $H M T$ genes}

In this study, we reconstructed the phylogenetic trees of the $C G S$ and $M M T$ genes in plants to understand their evolutionary history. The genes from representative whole-genome plants lineages, which contained monocots, eudicots, basal angiosperms, gymnosperms, basal land plants and chlorophyta, were surveyed (Tables S1,S2 in File S3). The phylogenetic trees were constructed using maximum likelihood (ML) and Neighbor-joining (NJ) methods (File S1). Due to the similar topologies of ML and NJ trees, ML trees were shown with higher support values

194 (Fig. 1, Figs. S1A-B in File S2).

First, the $C G S$ genes were widely separated in plant lineages from algae to angiosperms. In total, 49 representative $C G S$ s were used to reconstruct the phylogenetic tree, and the $C G S$ genes in algae, as the outgroup, were located at the base. The $C G S$ genes in gymnosperms and basal land plants had not diverged and were grouped in Class 3, while the CGSs had diverged into two classes in angiosperms, Class 1 and Class 2 (Fig. 1, Fig. S1A in File S2). In Class 1, all of the genes were contained in angiosperms, whereas, in Class 2, only the genes contained in grasses were present. The results indicated the $C G S$ genes might have diverged asynchronously in angiosperms.

203 However, MMT genes were not found in algae, which appeared from the ancestor of land plants. Furthermore, $M M T$ genes were relatively conserved, with only one or two copies, except for PpaMMTs with 3 copies. In total, 43 MMTs were surveyed. Phylogenetically, the evolutionary relationship of $M M T$ genes with their species relationship was relatively consistent (Fig. 1, Fig. S1B in File S2). As for the HMT genes, we have reported that they existed in kinds of plant lineages, and they have diverged into two classes in all of the seed plants (Fig. 1) (Zhao et al., 2018). Therefore, the phylogenetic relationships between $C G S, M M T$ and $H M T$ genes

210 showed that the MMTs were conserved, while the HMTs and CGSs had diverged in grasses and

211 all of the seed plants, respectively. The results implied that these genes have experienced

212 asynchronous divergence during their evolution.

\section{Intron-exon structures of $C G S s, M M T s$ and $H M T s$}


214

215

216

217

218

219

220

221

222

223

224

225

226

227

228

229

230

231

232

233

234

235

236

237

238

239

240

241

242

243

244

The divergence of genes is partly reflected in their structures. The intron-exon structures, as well as the number, length and position of introns and exons, in the $C G S, M M T$ and $H M T$ genes were analyzed (Table S3 in File S3).

The analysis of the number and position of introns and exons showed that most CGS genes in land plants contained an 11-exon and 10-intron pattern (Table S3A in File S3). For example, in Class 1, $26(26 / 35=74.3 \%)$ genes maintained the pattern and the remaining 9 had different degrees of intron gains or losses. In Class 3 all of the CGSs belonged to the 11-exon and 10intron pattern, except for the unknown PglCGS and PsiCGS. However, in Class 2 the number of exons was less than 6 and half of them $(5 / 10=50 \%)$ contained only 2 exons. The results showed that the divergence in exon-intron numbers have occurred between Class 2 and other classes in CGS genes. In addition, $76.2 \%(32 / 42=76.2 \%)$ of $M M T$ genes contained a 12-exon and 11intron pattern, while the remaining $23.8 \%(10 / 42=23.8 \%)$ experienced intron gain or loss events of different degrees (Table S3B in File S3). Generally, the exon-intron pattern of MMT genes was conserved during evolution, which was similar with HMT genes $(70.67 \%$ HMTs had a 7exon and 6-intron pattern) (Zhao et al., 2018).

Besides the number and position, the length of exons and introns was also considered in our study. In the three gene families, the corresponding lengths of exons were basically consistent, except for the $C G S$ genes in Class 2, while the corresponding lengths of introns were various in all of the genes (Table S3 in File S3). Finally, the analysis of the exon-intron structures indicated that the structures of the HMT and MMT genes were conserved, while the intron-exon numbers in $C G S$ genes diverged, especially in Class 2.Protein motifs analysis in MEME

Protein structures were analyzed to survey the conserved protein motifs of CGSs, MMTs and HMTs in MEME. In total, 16 motifs were identified and shown in CGSs (Fig. 2, Fig. S2A in File S2). Among them, 12 motifs (motif 1 to motif 6 and motif 9 to motif 14), located in the middle and C-terminal of the CGS proteins, were found in all CGS proteins (Fig. 2, Fig. S2A in File S2). However, the motifs in N-terminals, such as motif 7, 8, 15 and 16, were divided among classes. For instance, motifs 7, 8 and 16 were in Class 1 and Class 3, while motif 15 was in Class 2. The MTO1 region is essential for the negative feedback regulation of $C G S$ genes, which is located in the N-terminals of CGS (marked in red lines in Fig. 3 and File S1). In this study, the MTO1 region was only found in motif 8. Hence, the CGSs in Class 2 had lost their MTO1 regions during evolution (Fig. 3). In addition, some CGSs in Class 1, such as AthCGS2 and 
$245 \mathrm{BraCGS2}$, lost their MTO1 regions. Furthermore, three key sites in the MTO1 region (R77, S81

246 and G84 in AtCGS1) were not detected in Class 2, AthCGS2 or BraCGS2. In view of the

247 functions of the MTO1 region, the results indicated that the negative feedback regulation might

248 have been lost in Class 2, AthCGS2 and BraCGS2.

249 However, in MMT proteins, 16 motifs were totally consistent in all of the MMTs, except

250 BdiMMT1 and RcoMMT, which indicated that the protein motifs of MMTs were conserved

251 during evolution (Fig. 4, Fig. S2B in File S2). Similarly, the protein motifs of HMTs were also

252 conserved (Zhao et al., 2018). Based on the results above, the divergence of protein motifs has

253 occurred in CGS proteins, but not in HMTs and MMTs.

254 The selection pressure of the $C G S, H M T$ and $M M T$ family

255 Selection pressure is used to identify the genes have undergone adaptive evolution. To

256 analyze the selection pressure of the gene families, the $\omega$ values $(\omega=d N / d S)$ were estimated, and

257 the $\omega$ value was defined as the ratio of nonsynonymous and synonymous substitution. The

258 results showed that the $\omega$ values of CGSs, MMTs and HMTs were 0.19, 0.17 and 0.16,

259 respectively. The selection pressures showed that they were under stringent negative selection

260 during evolution, and hence their functions were stringent conserved during evolution.

261 qRT-PCR analysis of $C G S, M M T$ and $H M T$ genes in soybean

262 The expression of genes could reflect their functional divergence to some extents. To verify

263 their expression patterns, we analyzed the expression of CGSs, MMTs and HMTs in soybean (Fig.

$2645 \mathrm{~A}-\mathrm{C})$. In this study, the organs of leaves, stems, flowers and $2 \mathrm{w}-6 \mathrm{w}$ pods were collected and 265 analyzed.

266 The expression patterns of GmaCGS1 and GmaCGS2 were similar. Both of them were

267 highly expressed in leaves and flowers, but significantly decreased during the development

268 process of pods (Fig. 5A). Similarly, the expression models of GmaMMTs were analogous,

269 significantly highly expressed in stems, leaves, flowers and 2-week pods, and gradually

270 decreased during the development of pods (Fig. 5B). However, in GmaHMTs, the expression

271 patterns were varied (Fig. 5C). For example, the expression of GmaHMT1 and GmaHMT3 was

272 significantly higher in the pods and flowers than in the leaves and stems. On the contrary, the

273 GmaHMT2 was fluctuant in different organs, such as leaves, stems, flowers and pods, and the

274 expression levels of GmaHMT4 were significantly higher in leaves than in flowers, stems and

275 different pods. Above all, the expression patterns within GmaCGSs or GmaMMTs were 
276 consistent, respectively, yet the GmaHMTs were distinct from each other. The different

277 expression patterns of the three gene families might be essential to supply methionine for the 278 growth and development of soybeans.

279 Expression profiles of CGSs, HMTs and MMTs in PLEXdb

280 To further investigate the gene expression patterns, the tissue expression profiles of CGSs, 281 HMTs and MMTs were widely analyzed in Arabidopsis, soybean and rice in the PLEXdb 282 database (Fig. S3A-C in File S2). First, the expression patterns of CGSs were analyzed. In

283 Arabidopsis, both AthCGS1 and AthCGS2 were fluctuant in all of the tissues, but their expression 284 levels were generally higher in vegetative tissues than in productive tissues (Fig. S3A in File S2). 285 Nevertheless, the expression intensity of AthCGS1 (11-14) and AthCGS2 (3-7) was different. In 286 soybean, only GmaCGS1 was detected in the database. The expression trends of GmaCGS1 were 287 similar in the qRT-PCR results, and it was highly expressed in leaves but gradually decreased in 288 seeds and pods (Fig. S3B in File S2). Notably, the expression of OsaCGSs was varied. For 289 instance, the expression of OsaCGSI was fluctuant in vegetative and productive tissues. The 290 OsaCGS3 was highly expressed in vegetative tissues, such as leaves, roots and seedlings, while 291 the OsaCGS5 was higher in endosperms than in vegetative tissues. It was worth noting that their 292 expression intensities were also different, and the highest was found in OsaCGS3 (intensity from 29311 to 14), followed by OsaCGS1 (intensity from 5 to 8) and OsaCGS5 (intensity from 1 to 4). 294 In $M M T$ genes, the expression of OsaMMT was not detected (Fig. S4A,B in File S2). In 295 Arabidopsis, the expression levels of AthMMT were basically stable in different tissues, except 296 for seeds. In seeds, the expression level of AthMMT was lower than in other vegetative and 297 productive tissues (Fig. S4A in File S2). In soybean, the expression of GmaMMT1 was high in 298 vegetative tissues and the early stage of seeds, but low in fully grown pods. However, the 299 GmaMMT2 in different tissues was relative stable compared with GmaMMT1 (Fig. S4B in File 300 S2). The expression of HMTs in the database has also been comprehensively analyzed in our 301 previous article (Zhao et al., 2018). Their expression patterns were various, which have been 302 confirmed by the qRT-PCR results in this study. Some HMTs were widely expressed in different 303 tissues, while others were particularly highly expressed in specific tissues, such as seeds or

304 leaves. It is worth noting that the expression divergence of HMTs was not clade-specific.

305 Generally, the expression of the three key enzymes of CGS, HMT and MMT has experienced 306 varying degrees of divergence. 
307 Promoter analysis of $C G S$ s, $H M T$ s and $M M T$ s in soybean, Arabidopsis and rice

308 To understand the expression regulation and divergence, the promoters of CGSs, HMTs and

309 MMTs were examined and the cis-acting regulatory elements were predicted in silico. A global

310 analysis of regulatory elements in the promoters of CGSs, HMTs and MMTs in soybean,

311 Arabidopsis and rice are shown in Table S4 in File S3. In this study, we divided the motifs into 2

312 groups: Group 1 (related to levels and locations of expression) and Group 2 (related to responses

313 to stresses) (Table S4 in File S3).

314 First, the numbers of motifs of GmaCGS in Group 1 and Group 2 were similar. However,

315 unlike GmaCGS2, GmaCGS1 had two specific motifs, a 5UTR Py-rich stretch and TA-rich

316 region, related to high expression levels, which indicated that the expression levels of GmaCGS1

317 might be higher than GmaCGS2 (Table S4A in File S3). As for AthCGSs, the numbers of motifs

318 in the two groups were different. In Group 1, AthCGS2 (10 motifs) had more motifs than

319 AthCGS1 (5 motifs), but the opposite was the case in Group 2. Considering their similar spatio-

320 temporal expression patterns, the differences in Group 2 might suggest differences in their

321 responses to different stresses (Table S4A in File S3). In rice, the OsaCGSs were divided into

322 two classes, OsaCGS1-4 in Class 2 and OsaCGS5 in Class 1. OsaCGSs in Class 2 (19 in

323 OsaCGS1, 24 in OsaCGS2, 23 in OsaCGS2 and 19 in OsaCGS2) had more elements responsive

324 to stresses than OsaCGS5 (6 elements), suggesting that the OsaCGSs in Class 2 might have an

325 important role in responses to stresses. In view of expression levels, OsaCGS2, OsaCGS4 and

326 OsaCGS5 had one 5UTR Py-rich stretch, and OsaCGS3 had one TA-rich region. In our study,

327 the expression intensity of OsaCGS3 was higher than that of OsaCGS1 and OsaCGS5, which

328 indicated that the TA-rich region might be necessary to the high expression levels in OsaCGSs

329 (Table S4A in File S3). The MMTs were relatively conserved, with one or two copies. For

330 example, in rice and Arabidopsis, there was only one copy. However, in soybean, there were two

331 copies, and there was a greater number of motifs of GmaMMT2 than of GmaMMT1 in Group 1

332 (18) and Group 2 (16) (5 and 8, respectively) (Table S4B in File S3). Moreover, in GmaMMT2,

333 there were 15 enhancers in the promoter, which might be the reason why the expression intensity

334 of GmaMMT2 was higher than that of GmaMMT1.

335 As for HMTs, the AthHMTs have been analyzed in our previous study (Table S4C in File

336 S3). In Group 1 and Group 2, the motifs of AthHMTs were different. In soybean, there was a

337 greater number of motifs of GmaHMT4 than of GmaHMT1-3 in Group 1 (25) (5, 7 and 1, 
338 respectively), while in Group 2, there were fewer motifs of GmaHMT4 (7) than the others (14,

33930 and 15, respectively). Similarly, the motifs of OsaHMTs were varied in Group 1 and Group 2

340 (Table S4C in File S3). Therefore, just as their expression patterns were distinct, their promoters

341 were varied.

342

343 DISCUSSION

344 The divergence of $C G S, H M T$ and $M M T$ genes was asynchronous.

$345 C G S, M M T$ and HMT genes are vital to the synthesis of methionine in plants (Datko et al.,

346 1974; Bourgis et al., 1999; Ranocha et al., 2001; Lee et al., 2008; Cohen et al., 2017a). In this

347 study, their evolutionary histories were reconstructed. Their phylogenetic relationships were

348 different, in which the MMTs were conserved during evolution, yet the $C G S$ and $H M T$ gene

349 families in grasses and seed plants diverged in varying degrees. Similarly, the gene structures of

350 the MMTs and HMTs were conserved, but the structure of CGSs diverged in the N-terminals and

351 intron-exon numbers. Further, the divergence in the N-terminals and intron-exon structure in

$352 C G S$ s was mainly present in Class 2. Therefore, the evolution of CGS, HMT and MMT gene

353 families was asynchronous.

354 Although varying degrees of divergence has been detected in CGSs, HMTs and MMTs, they

355 were all under stringent negative selection pressures. The results indicated that the three families

356 did not undergo adaptive evolution. However, a partial subfunctionalization might have occurred.

357 Subfunctionalization in evolution often results from changes in gene expression (Gallego-

358 Romero et al., 2012; Wang et al., 2012). In our previous results, the subfunctionalization of

359 HMTs has occurred in their expression, which might be vital to supplying methionine for the

360 development seeds and growth of plants (Zhao et al., 2018). However, MMTs were similar in

361 their expression patterns. Nevertheless, the expression patterns of CGS genes in dicots were also

362 basically consistent. However, in rice, the expression of CGS genes was varied. The OsaCGSs in

363 Class 2 lost their MTO1 region. In view of the functions of the MTO1 region, which destabilizes

364 the $C G S$ mRNA, it seemed that the expression of the OsaCGSs might be influenced by the loss

365 of the MTO1 region (Chiba et al., 1999). Furthermore, according to the analysis of promoters in

$366 C G S$ genes, the OsaCGSs without its MTO1 region were rich in the motifs related to stress

367 responses (Table S4A in File S3). The results seemed like that the divergence of OsaCGSs in

368 Class 2 might be related to its response to different stresses in rice. However, notably, the similar 
369 expression divergence did not occur between AthCGSs, although AthCGS2 has also lost its

370 MTO1 region. Moreover, the numbers of stress response motifs in AthCGS2 were fewer than in

371 AthCGS1. Thus, it seemed that the expression divergence of CGSs in grasses and dicots might be

372 independent of the loss of the MTO1 region, or the MTO1 region has a different impact on the

373 grasses and dicots, which need to be further studied. In any case, the CGSs, HMTs and MMTs

374 genes have experienced inconsistent divergence in evolution and expression.

375 Evolution pattern of Met biosynthetic pathway in plant lineages.

376 Gene duplication is of huge significance for the evolution of metabolic pathways. The

377 production of gene duplication, two or more copies of genes, leads to the increase of genome size,

378 diversification of enzymes and supplies the raw materials for new properties (Fondi et al., 2007;

379 Lynch \& Conery, 2000; Zhang, 2003). Functional innovations in evolution often result from the

380 expressional changes of duplicated genes (Gallego-Romero et al., 2012; Wang et al., 2012). In

381 our study, the CGS, HMT and MMT genes have been duplicated and diverged during evolution.

382 In addition, previous research has also proposed that the evolution and divergence of metabolic

383 pathways may be disclosed by comparing the sequence and the structure of genes of the same

384 and different routes from organisms (Fondi et al., 2007; Goolsby et al., 2018).Therefore,

385 combining the evolutionary and expressional pattern of CGS, HMT and MMT genes, we

386 proposed the evolutionary and functional models of Met biosynthetic pathway in plant lineages

387 (Fig. 6).

388 In algae, only $C G S$ and $H M T$ genes were found, which suggested that the methionine in

389 algae was only synthesized by the de novo Asp family pathway. As for HMT genes, Bradbury

390 has reported that the functions of $H M T$ genes contained the ancient, repair of $(R, S)$-AdoMet, and

391 the acquired, SMM catabolism (Bradbury et al., 2014). Therefore, in algae, the HMT genes might

392 be mainly involved in the repair of $(R, S)$-AdoMet. Afterwards, land plants began to appear in the

393 world. The evolutionary history of MMTs suggested that MMTs might occur in the ancestor of

394 land plants. In basal land plants, such as moss, Selaginella moellendorffii and so on, the CGSs,

$395 H M T$ s and MMTs were grouped together during evolution, respectively, which indicated that the

396 three gene families might not be divergent. Therefore, in basal land plants, the methionine was

397 supplied to the whole plants by the Asp family pathway and the SMM cycle together. However,

398 due to the loss of expression data in this study, their specific functional patterns were unknown. 
In seed plants, different divergence has occurred in the three gene families. First, the $M M T$

400

401

402

403

404

405

406

407

408

409

410

411

412

413

414

415

416

417

418

419

420

421

422

423

424

425

426

427

428

429

and $C G S$ genes had a high and low expression in vegetative tissues and reproductive tissues, respectively. The high expression of MMTs and CGSs in vegetative tissues suggested that the Asp family pathway probably supplies the methionine largely during the early vegetative growth of seed plants, which is consistent with Cohen's results (Cohen et al., 2017b). It is noteworthy that $H M T$ genes have diverged into two clades in seed plants, and their expression has obviously experienced divergence. In the two clades, the AtHMT1 and AtHMT3, and GmaHMT1-3s were primarily functioned in seeds, while AtHMT2 and GmaHMT4 were largely functioned in leaves or stable in all tissues. The results indicated that more HMTs functioned in seeds in Arabidopsis and soybean than the MMTs. Finally, by combining the evolution and expression of CGS, $H M T$ and MMT genes together, we inferred their co-functional models in seed plants as follows (Fig. 5). In vegetative tissues, e.g. leaves, an amount of Met is synthesized, mainly by CGS through the Asp family pathway. Next, a considerable amount of Met enters into the SMM cycle, in which the Met is converted into SMM by MMT. Subsequently, the SMMs are transfered into reproductive tissues (seeds) through phloems (Bourgis et al., 1999; Ranocha et al., 2001; Lee et al., 2008; Cohen et al., 2017b). In the meantime, some SMMs are reconverted into the SMM cycle in leaves. In the seeds, the transported SMMs are reconverted into Met by HMTs. This is the main way through which Met is supplied for seed development, especially in the late stage of seed development (Cohen et al., 2017b). Furthermore, through the Asp family pathway, the methionine is synthesized for seed development (Cohen et al., 2017b). It is noteworthy that regardless of the tissues, $R, S$-SAMs are always recovered by HMTs (Bradbury et al., 2014). Therefore, adequate methionine is supplied for the growth and development of seed plant through the synergistic function of CGSs, HMTs and MMTs.

\section{CONCLUSIONS}

In the present study, the three key enzymes of CGS, MMT and HMT in the biosynthesis of Met were investigated in detail. The evolution patterns of the three gene families have undergone divergence: MMTs were conserved, while CGSs and HMTs have diverged in the grasses or all of the seed plant. The gene structures were conserved, except for CGS genes in Class 2. For gene expression, similar as their evolutionary pattern, the $M M T$ s were conserved, and the $C G S \mathrm{~s}$ and HMTs diverged among tissues. Furthermore, the functions of HMTs were diverged into the 
430 repair of $(R, S)$-AdoMet, and SMM catabolism. Therefore, subfunctionalization has occurred in 431 both $C G S$ and HMT gene families. Finally, based on the evolution and expression divergence of $432 C G S \mathrm{~s}, H M T \mathrm{~s}$ and $M M T \mathrm{~s}$, we built the evolution model of Met biosynthetic pathway in plants, 433 which is basically consistent with the evolution of the plants. The model also reveals that CGSs, 434 HMTs and MMTs are essential to supply the methionine for the growth and development of 435 different plant lineages.

436

437

\section{Supplementary information}

439 File S1: The amino acids alignments of CGSs and MMTs used in the study.

440 File S2:

441 Figure S1. Phylogenetic tree of the $M M T(\mathrm{~A})$ and $C G S(\mathrm{~B})$ gene family in plants. The trees were 442 constructed with maximum likelihood (ML) and neighbor-joining (NJ) methods based on the 443 amino acid (aa). Support values ( $>50 \%$ of ML and NJ) for the two trees are shown on the 444 branches, respectively. The black star indicates the divergence of Class 1 and Class 2. Gene 445 names and identifiers are shown in Table S1 in File S3.

446 Figure S2. Sequences of the conserved motifs detected by the MEME analysis on the CGS (A) 447 and MMT (B) homologs across plants. The height of the each letter denotes the probability of the 448 letter at that position, and total height of the stack represents the information content of that 449 position.

450 Figure S3A. Tissue-specific expression data of $A t h C G S$ s from PLEXdb. The green, red and 451 orange boxes represent vegetative tissues such as roots, stems and leaves, flowers and seeds, 452 respectively.

453 Figure S3B. Tissue-specific expression data of GmaCGSs from PLEXdb. The green and blue 454 boxes represent root apical meristem and leaf non-meristem. The pink, orange, red and purple 455 boxes represent bean $2 \mathrm{~mm}$, bean $5 \mathrm{~mm}$, pod elongation and fully grown, respectively.

456 Figure S3C. Tissue-specific expression data of OsaCGSs from PLEXdb. The pink, yellow, 457 green, orange and red boxes represent emb6D, endo6D, leaf, root and seedling, respectively.

458 Figure S4A. Tissue-specific expression data of AthMMTs from PLEXdb. The green, red, orange 459 boxes represent vegetative tissues such as roots, stems and leaves, flowers, and seeds, 460 respectively. 
461 Figure S4B. Tissue-specific expression data of GmaMMTs from PLEXdb. The green and blue 462 boxes represent root apical meristem and leaf non-meristem. The pink, orange, red and purple 463 boxes represent bean $2 \mathrm{~mm}$, bean $5 \mathrm{~mm}$, pod elongation and fully grown, respectively.

464 File S3:

465 Table S1. List of $C G S(\mathrm{~A})$ and $M M T(\mathrm{~B})$ sequences used in this study.

466 Table S2. Distribution of CGS (A) and $M M T(\mathrm{~B})$ genes in different classes.

467 Table S3. Size variations of exons and introns in $C G S(\mathrm{~A})$ and $M M T(\mathrm{~B})$ genes.

468 Table S4. Analysis of the motifs in the promoters of CGSs (A), MMTs (B) and HMTs (C).

469 Table S5. Primers used in this study.

470

471 REFERENCES

472 Bailey TL, Bodén M, Buske FA, Frith M, Grant CE, Clementi L, Ren J, Li WW, Noble WS. 473 2009. MEME SUITE: tools for motif discovery and searching. Nucleic Acids Research 37:202474208.

475 Bolten CJ, Schroder H, Dickschat J, Wittmann C. 2010. Towards methionine overproduction 476 in Corynebacterium glutamicum - methanethiol and dimethyldisulfide as reduced sulfur sources. 477 Journal of Microbiology and Biotechnology 20(8):1196-1203.

478 Bourgis F, Roje S, Nuccio ML, Fisher DB, Tarczynski MC, Li C, Herschbach C, 479 Rennenberg H, Pimenta MJ, Shen TL, Gage DA, Hanson AD. 1999. S-methylmethionine 480 plays a major role in phloem sulfur transport and is synthesized by a novel type of 481 methyltransferase. The Plant Cell 11:1485-1498.

482 Bradbury LM, Ziemak MJ, Elbadawi-Sidhu M, Fiehn O, Hanson AD. 2014. Plant-driven

483 repurposing of the ancient S-adenosylmethionine repair enzyme homocysteine S-

484 methyltransferase. Biochemistry Journal 463, 279-286.

485 Chiba Y, Ishikawa M, Kijima F, Tyson RH, Kim J, Yamamoto A, Nambara E, Leustek T, 486 Wallsgrove RM, Naito S. 1999. Evidence for autoregulation of cystathionine $\gamma$-synthase mRNA 487 stability in Arabidopsis. Science 286:1371-1374.

488 Cohen H, Hacham Y, Panizel I, Rogachev I, Aharoni A, Amira R. 2017b. Repression of 489 CYSTATHIONINE $\gamma$-SYNTHASE in seeds recruits the S-methylmethionine cycle. Plant 490 Physiology 174:1322-1333.

491 Cohen H, Israeli H, Matityahu I, Amir R. 2014. Seed-specific expression of a feedback- 
492 insensitive form of CYSTATHIONINE- $\gamma$-SYNTHASE in Arabidopsis stimulates metabolic and

493 transcriptomic responses associated with desiccation stress. Plant Physiology 166:1575-1592.

494 Cohen H, Salmon A, Tietel Z, HachamY, Amir R. 2017a. The relative contribution of genes

495 operating in the S-methylmethionine cycle to methionine metabolism in Arabidopsis seeds. Plant 496 Cell Reports 36:731-743.

497 Cohen H, Shir OM, Yu Y, Hou W, Sun S, Han T, Amir R. 2016. Genetic background and

498 environmental conditions drive metabolic variation in wild type and transgenic soybean (Glycine 499 max) seeds. Plant Cell and Environment 39:1805-1817.

500 Dash S, Van Hemert J, Hong L, Wise RP, Dickerson JA. 2012. PLEXdb: gene expression 501 resources for plants and plant pathogens. Nucleic Acids Research 40(D1):D1194-D1201.

502 Datko AH, Giovanelli J, Mudd SH. 1974. Homocysteine biosynthesis in green plants: O-

503 Phosphorylhomoserine as the physiological substrate for cystathionine $\gamma$-synthase. The Journal

504 of Biological Chemistry 249 (4):1139-1155.

505 Ferla MP, Patrick WM. 2014. Bacterial methionine biosynthesis. Microbiology 160:1571-1584.

506 Fondi M, Brilli M, Emiliani G, Paffetti D, Fani R. 2007. The primordial metabolism: an

507 ancestral interconnection between leucine, arginine, and lysine biosynthesis. BMC Evolutionary

508 Biology 7(Suppl 2):S3.

509 Galili G, Amir R. 2013. Fortifying plants with the essential amino acids lysine and methionine

510 to improve nutritional quality. Plant Biotechnology Journal 11:211-222.

511 Gallego-Romero I, Ruvinsky I, Gilad Y. 2012. Comparative studies of gene expression and the

512 evolution of gene regulation. Nature Reviews 13:505-516.

513 Goolsby EW, Moore AJ, Hancock LP, De Vos JM, Edwards EJ. 2018. Molecular evolution

514 of key metabolic genes during transitions to C4 and CAM photosynthesis. American Journal of

515 Botany 105(3): 1-12.

516 Guindon S, Gascuel O. 2003. A simple, fast, and accurate algorithm to estimate large

517 phylogenies by maximum likelihood. Systematic Biology 52:696-704.

518 Hall TA. 1999. BioEdit: A user-friendly biological sequence alignment editor and analysis

519 program for Windows 95/98/NT. Nucleic Acids Symposium Series 41:95-98.

520 Hanafy MS, Rahman SM, Nakamoto Y, Fujiwara T, Naito S, Wakasa K, Ishimoto M. 2013.

521 Differential response of methionine metabolism in two grain legumes, soybean and azuki bean, 522 expressing a mutated form of Arabidopsis cystathionine $\gamma$-synthase. Journal of Plant Physiology 
523

524

525

526

527

528

529

530

531

532

533

534

535

536

537

538

539

540

541

542

543

544

545

546

547

548

549

551

550 Nielsen NC, Dickinson CD, Cho TJ, Thanh VH, Scallon BJ, Fischer RL, Sims TL, Drews 552 Cell 1:313-328.

553 Ominato K, Akita H, Suzuki A, Kijima F, Yoshino T, Yoshino M, Chiba Y, Onouchi H,

170:338-345.

Harada JJ, Barker SJ, Goldberg RB. 1989. Soybean $\beta$-conglycinin genes are clustered in several DNA regions and are regulated by transcriptional and posttranscriptional processes. The Plant Cell 1:415-425.

Kim J, Lee M, Chalam R, Martin MN, Leustek T, Boerjan W. 2002. Constitutive overexpression of cystathionine $\gamma$-synthase in Arabidopsis leads to accumulation of soluble methionine and S-methylmethionine. Plant Physiology 128:95-107.

Kim J, Leustek T. 2000. Repression of cystathionine $\gamma$-synthase in Arabidopsis thaliana produces partial methionine auxotrophy and developmental abnormalities. Plant Science 151:918.

Kocsis MG, Ranocha P, Gage DA, Simon ES, Rhodes D, Peel GJ, Mellema S, Saito K, Awazuhara M, Li C, Meeley RB, Tarczynski MC, Wagner C, Hanson AD. 2003. Insertional inactivation of the methionine S-methyltransferase gene eliminates the S-methylmethionine cycle and increases the methylation ratio. Plant Physiology 131:1808-1815.

Lee M, Huang T, Toro-Ramos T, Fraga M, Last RL, Jander G. 2008. Reduced activity of Arabidopsis thaliana HMT2, a methionine biosynthetic enzyme, increases seed methionine content. The Plant Journal 54:310-320.

Lescot M, Déhais P, Thijs G, Marchal K, Moreau Y, Van de Peer Y, Rouzé P, Rombauts S. 2002. PlantCARE, a database of plant cis-acting regulatory elements and a portal to tools for in silico analysis of promoter sequences. Nucleic Acids Research 30(1):325-327.

Livak KJ, Schmittgen TD. 2001. Analysis of relative gene expression data using real-time quantitative PCR and the 2- $\Delta \Delta \mathrm{Ct}$ method. Methods 25:402-408.

Lynch M, Conery JS. 2000. The evolutionary fate and consequences of duplicate genes. Science 290: 1151-1155.

Matityahu I, Godo I, Hacham Y, Amir R. 2013. Tobacco seeds expressing feedbackinsensitive cystathionine $\gamma$-synthase exhibit elevated content of methionine and altered primary metabolic profile. BMC Plant Biology 13:206. 51 GN, Goldberg RB. 1989. Characterization of the glycinin gene family in soybean. The Plant 
554 Naito S. 2002. Identification of a short highly conserved amino acid sequence as the functional

555 region required for posttranscriptional autoregulation of the cystathionine $\gamma$-synthase gene in

556 Arabidopsis. The Journal of Biological Chemistry 277:36380-36386.

557 Ranocha P, Bourgis F, Ziemak MJ, Rhodes D, Gage DA, Hanson AD. 2000. Characterization

558 and functional expression of cDNAs encoding methionine-sensitive and -insensitive

559 homocysteine S-methyltransferases from Arabidopsis. The Journal of Biological Chemistry

560 275:15962-15968.

561 Ranocha P, McNeil SD, Ziemak MJ, Li C, Tarczynski MC, Hanson AD. 2001. The S-

562 methylmethionine cycle in angiosperms: ubiquity, antiquity and activity. The Plant Journal

563 25:575-584.

564 Ranocha P, McNeil SD, Ziemak MJ, Li C, Tarczynski MC, Hanson AD. 2001. The S-

565 methylmethionine cycle in angiosperms: ubiquity, antiquity and activity. The Plant Journal

566 25(5):575-584.

567 Roje S. 2006. S-Adenosyl-L-methionine: beyond the universal methyl group donor.

568 Phytochemistry 67:1686-1698.

569 Sauter M, Moffatt B, Saechao MC, Hell R, Wirtz M. 2013. Methionine salvage and S-

570 adenosylmethionine: essential links between sulfur, ethylene and polyamine biosynthesis.

571 Biochemical Journal 451:145-154.

572 Song S, Hou W, Godo I, Wu C, Yu Y, Matityahu I, Hacham Y, Sun S, Han T, Amir R. 2013.

573 Soybean seeds expressing feedback-insensitive cystathionine $\gamma$-synthase exhibit a higher content

574 of methionine. Journal of Experimental Botany 64:1917-1926.

575 Tamura K, Stecher G, Peterson D, Filipski A, Kumar S. 2013. MEGA6: Molecular

576 evolutionary genetics analysis version 6.0. Molecular Biology and Evolution 30:2725-2729.

577 Thompson GA, Datko AH, Mudd SH, Giovanelli J. 1982. Methionine biosynthesis in Lemna:

578 studies on the regulation of cystathionine $\gamma$-synthase, O-phosphohomoserine sulfhydrase, and O-

579 acetylserine sulfhydrase. Plant Physiology 69:1077-1083.

580 Thompson JD, Gibson TJ, Plewniak F, Jeanmougin F, Higgins DG. 1997. The CLUSTAL_X

581 windows interface: Flexible strategies for multiple sequence alignment aided by quality analysis

582 tools. Nucleic Acids Research 25:4876-4882.

583 Wang Y, Wang X, Paterson AH. 2012. Genome and gene duplications and gene expression

584 divergence: a view from plants. Annals New York Academy Sciences 1256:1-14. 
585 Willke T. 2014. Methionine production-a critical review. Applied Microbiology and

586 Biotechnology 98:9893-9914

587 Yang Z. 2007. PAML 4: a program package for phylogenetic analysis by maximum likelihood.

588 Molecular Biology and Evolution 24:1586-1591.

589 Zhang J. 2003. Evolution by gene duplication: An update. Trends in Ecology \& Evolution 590 18:292-298.

591 Zhao M, Chen P, Wang W, Yuan F, Zhu D, Wang Z, Ying X. 2018. Molecular evolution and 592 expression divergence of HMT gene family in plants. International Journal of Molecular 593 Sciences 19 (4). 


\section{Figure 1}

The diagrams of the evolutionary relationships of CGS, HMT and MMT gene families.

A-C represent MMT, CGS and HMT gene family, respectively. The diagrams were based on their phylogenetic tree in Figure S1 and Zhao et al. 2018. The red, blue, purple, green and orange triangles represent algae, basal land plants, basal angiosperms and gymnosperms, monocots and dicots, respectively.
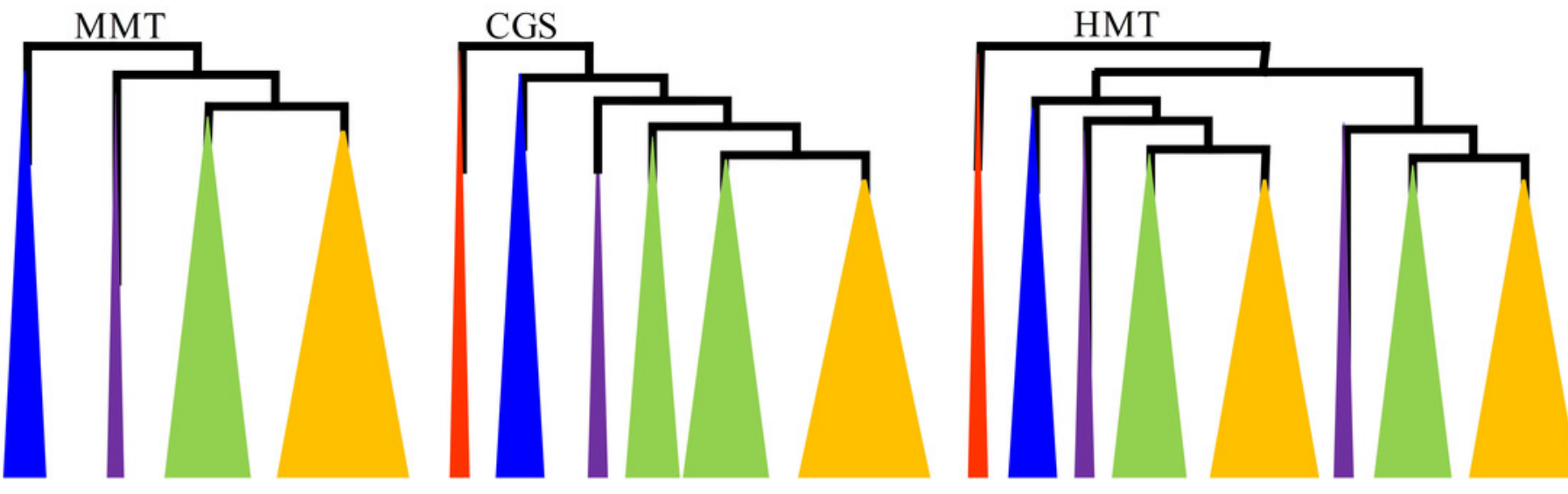


\section{Figure 2}

Conserved motifs of CGS proteins identified on the MEME analysis across plants.

Each motif is represented by a colored box numbered on the bottom. A-D represent Class 1 , Class 2, Class 3 and Outgroup, respectively. The amino acid sequences of these motifs are presented in Figure S1 in File2. The black lines represent unique sequences. The scale bar indicates number of amino acids. Names to the left indicate the clades to which the sequences belong to according to Figure S1 in File2.

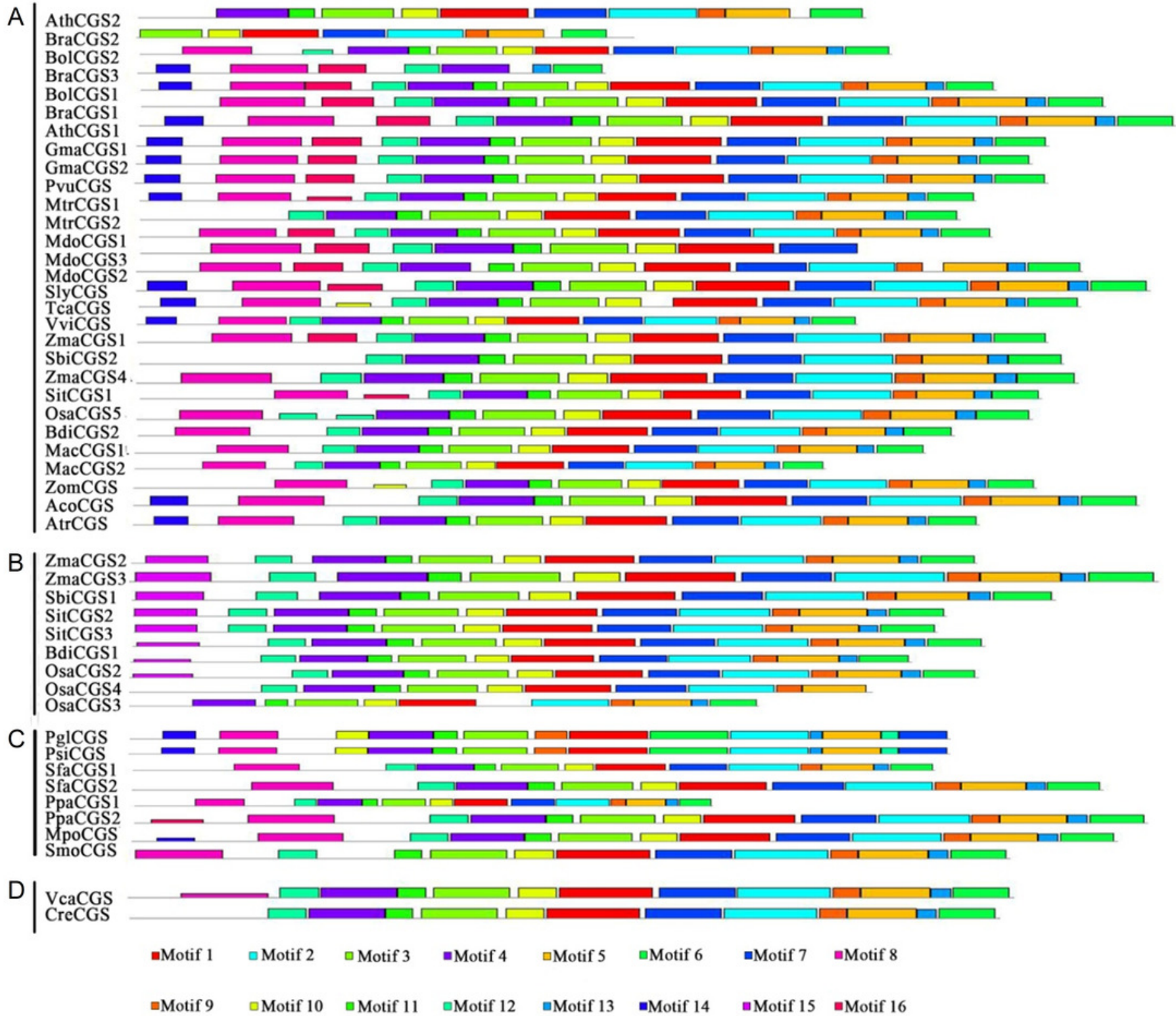


Figure 3

The sequence composition of the conserved regions in Class 1, Class 2 and Class 3 in CGS family.

A-C represent the conserved region in Class 1, Class 2 and Class 3, respectively. The MTO1 region positions are marked by red lines. The height of the each letter represents the probability of the letter at that position, and total height of the stack represents the information content of that position.
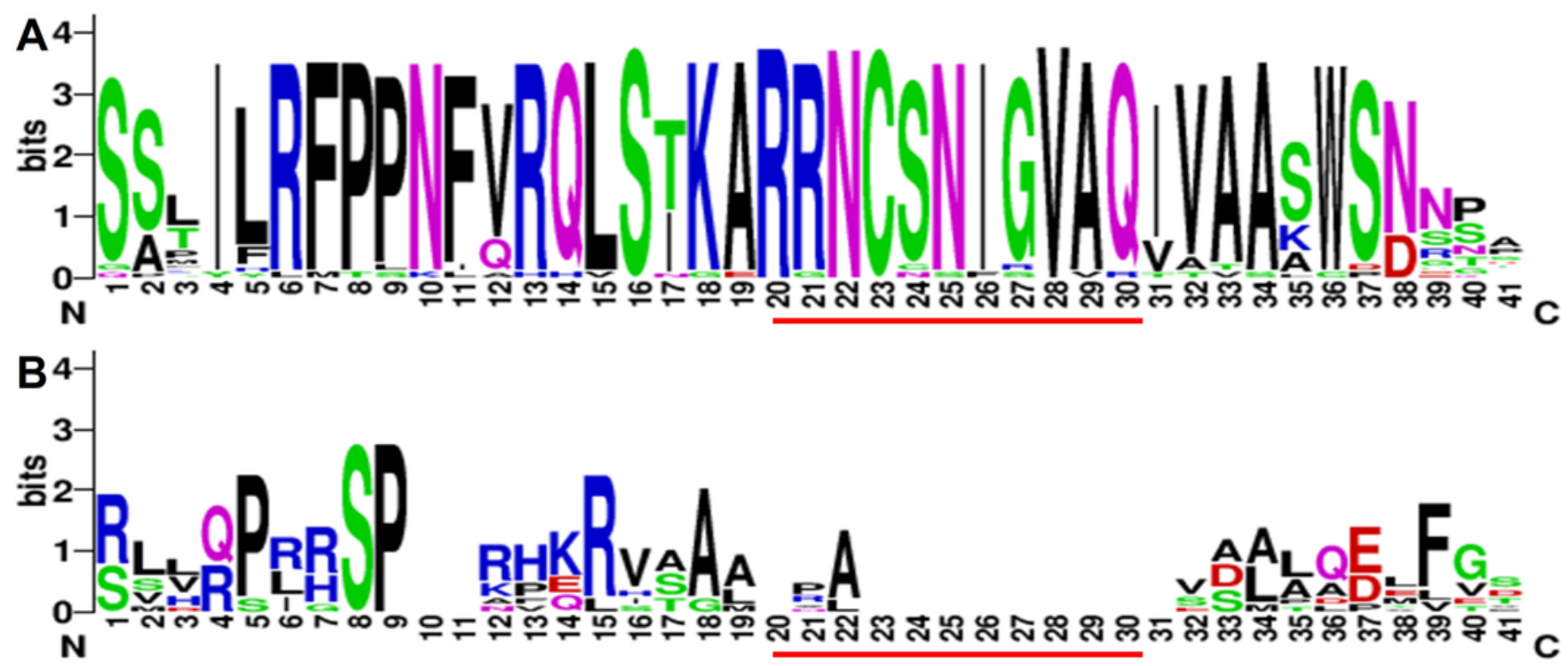

$\mathrm{C}_{4}-$
$3-$
은

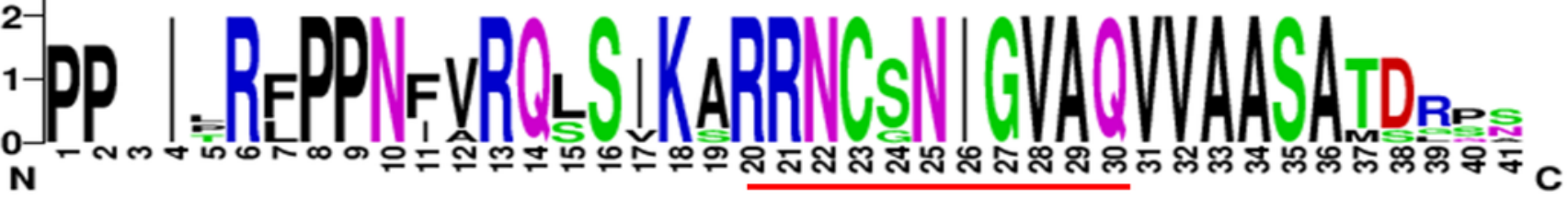


Figure 4

Conserved motifs of MMT proteins identified on the MEME analysis across plants.

Each motif is represented by a colored box numbered on the bottom. The amino acid sequences of these motifs are presented in Figure S1 in File2. The black lines represent unique sequences. The scale bar indicates number of amino acids. Names to the left indicate the clades to which the sequences belong to according to Figure S1 in File2.

\begin{tabular}{|l} 
MacMMT2 \\
MacMMT1 \\
OsaMMT \\
PviMMT2 \\
PviMMY1 \\
SitMMT \\
SbiMMT \\
ZmaMMT \\
BdiMMT3 \\
BdiMMT2 \\
BdiMMT1 \\
ZomMMT
\end{tabular}

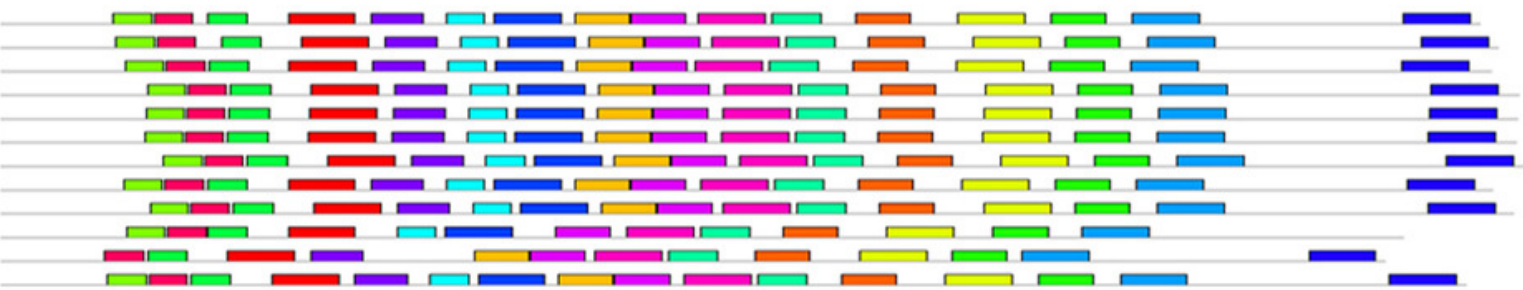

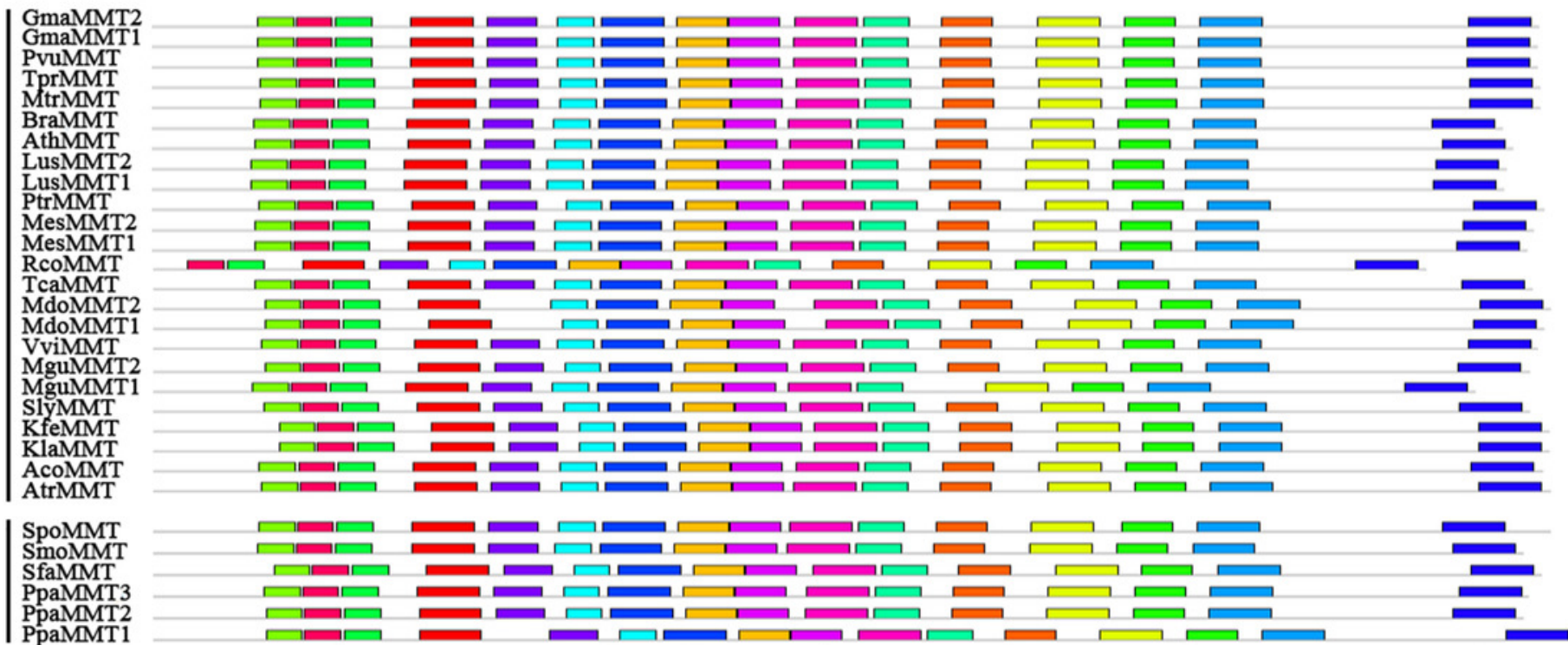
atif1
$\square$ Motif 2
$\square$ Motif 3
- Motif 4
$\square$ Motif 5
$\square$ Motif 6
- Motif 7
- Motif 8
Motif9
$\square$ Motif 10
Motif 11
$\square$ Motif12
ㅁ Motif 13
- Motif 14
— Motif 15
- Motif 16 


\section{Figure 5}

Expression of the GmaCGS, GmaMMT and GmaHMT genes during soybean development. 192.1528681021

A-H The spatio-temporal expression of GmaCGS1 (A) and GmaCGS2 (B), GmaMMT1 (C) and GmaMMT2 (D), and GmaHMT1 (E), GmaHMT2(F), GmaHMT3(G) and GmaHMT4(H). The total RNAs were isolated from stems, leaves of 14-day-old seedlings, flowers, and 2-, 4- and 6week-old pods after fertilization. The ACTIN gene was used as an internal control. The experiments were repeated using three independent biological samples. Error bar: standard deviation. The significance was tested in comparison with the expression of each gene in leaves. The * means significance at a $\mathrm{P}<0.05$ level, and the** represents the significance at a $\mathrm{P}<0.01$ level. 

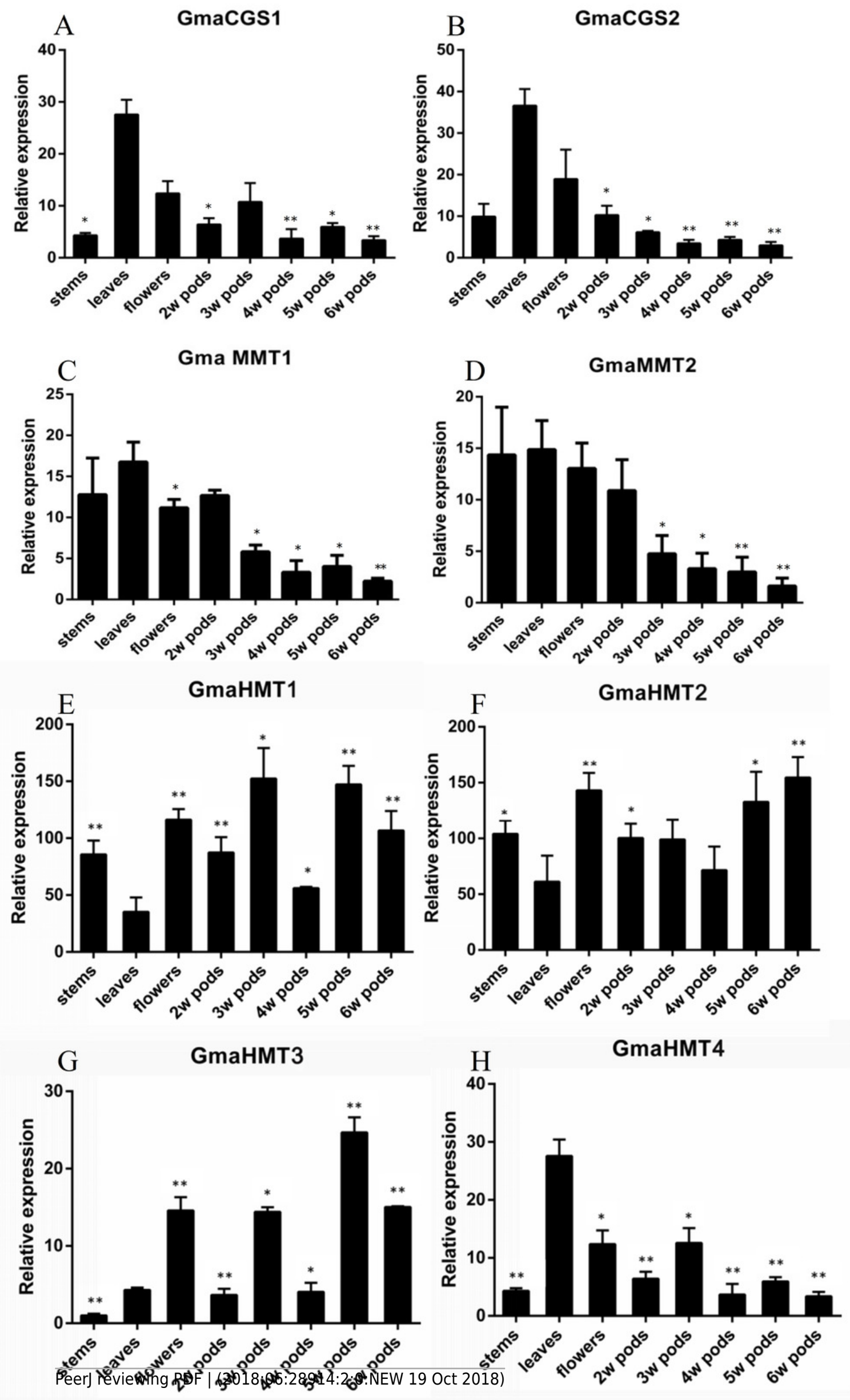


\section{Figure 6}

The functional model of CGSs, MMTs and HMTs to synthesize the methionine in plants.

The enzyme of CGS, MMT and HMT in aspartate family pathway and SMM were italics. Black arrows indicate the direction of evolution or flux of reaction. The green arrows indicate methionine flux during the growth and seeds development of seed plants. The thickness of green arrows indicates the strength of flow. CGS, cystathionine g-synthase; HMT, homocysteine S-methyltransferases; MMT, met S-methyltransferase; Met, Methionine; R,SSAM, R,S-adenosylmethionine; SMM, S-methyl-methionine.

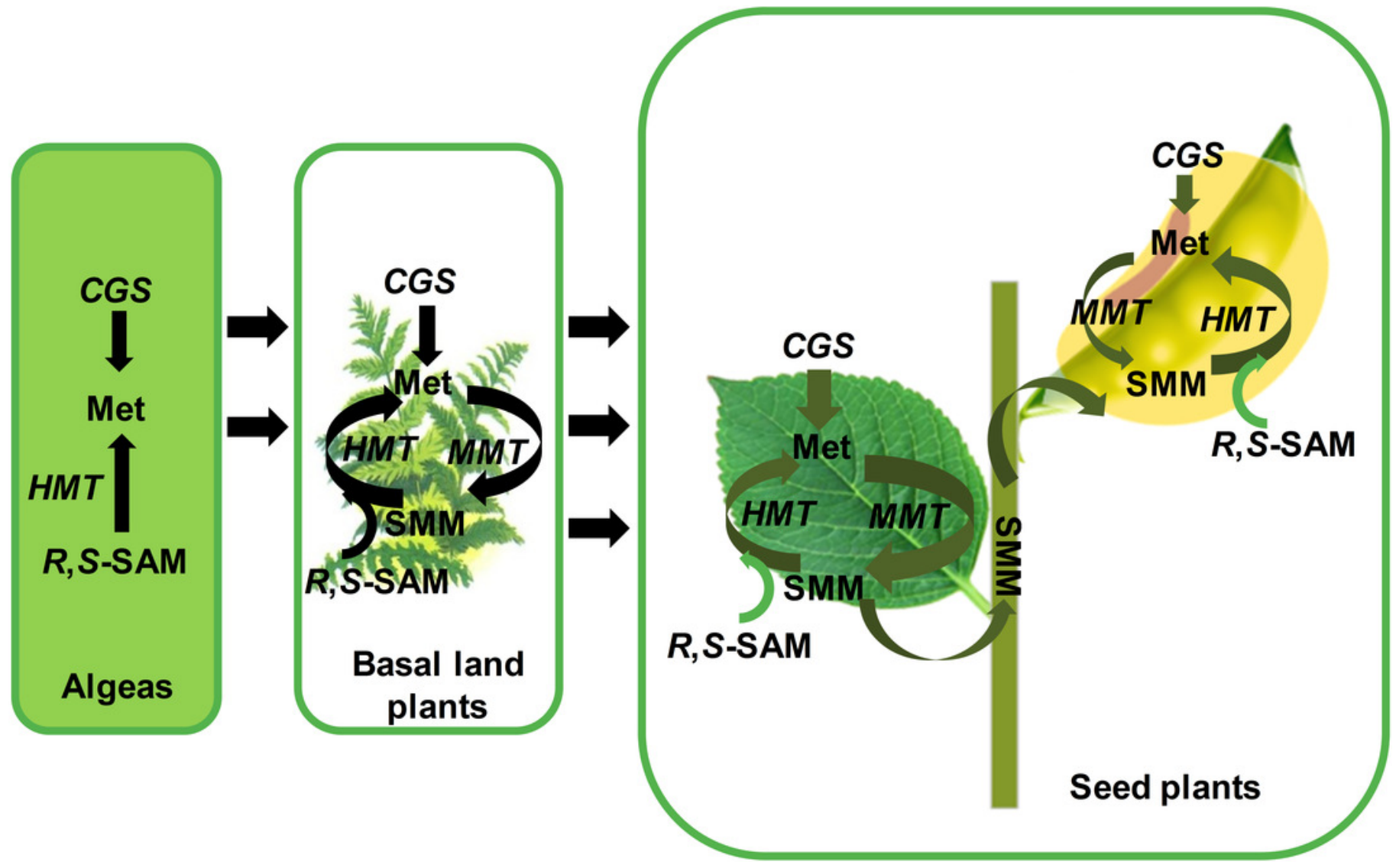

